\title{
REFLEXIONES SOBRE LA EDUCACIÓN SUPERIOR EN NUESTRO PAÍS
}

\author{
Álvaro Saieh*
}

* Miembro de la Junta Directiva y Presidente del Centro de Investigación en Economía y Finanzas (CIEF) de la Universidad Andrés Bello. Consejero de la Sociedad de Fomento Fabril (SOFOFA). 


\section{RESUMEN}

El artículo presenta argumentos en contra de la imposición de criterios rígidos, cuantitativos o cualitativos, para regular el desarrollo de la educación superior. Se sostiene que quienes demandan servicios educacionales parecen utilizar información más completa que la oficialmente disponible, siendo capaces de reconocer la diversidad de contenidos tras carreras o programas aparentemente comparables. En cuanto a los contenidos en la educación superior, se discute un sentido deseable para la evolución del sistema universitario chileno desde su actual estructura profesionalizante. Finalmente se postula que el apoyo estatal al sistema de educación superior debe ir desde los mecanismos actuales de apoyo directo o indirecto a las universidades hacia mecanismos de garantías privadas y estatales a préstamos a los alumnos de dicho nivel educativo.

\section{ABSTRACT}

This paper argues against imposing rigid quantitative or qualitative criteria to regulate the development of higher education. It holds that individuals who demand educational services appear to use more complete information than officially available data and that they are capable of recognizing the diversity of contents underlying apparently comparable study programs. Regarding contents in higher education, the article discusses a desirable direction for the evolution of Chile's higher education system from its current structure for professionalization. The article holds that coverage of government support for the higher education system needs to range from current mechanisms of direct and indirect support for universities to mechanisms of private and state guarantees and loans to students in higher education. 


\section{REFLEXIONES SOBRE LA EDUCACIÓN SUPERIOR EN NUESTRO PAÍS}

\section{INTRODUCCIÓN}

Aprecio que nuestro país ha dado pasos gigantescos para permitir la creación de nuevas instituciones de educación superior y para incorporar a los estudiantes, como parte activa de esa área, en la definición de qué carreras se ofrecen, con qué contenidos y en qué nivel de calidad educacional. Esto ha permitido un enorme y positivo desarrollo de la educación superior en el país. En la actualidad más de 220 entidades de educación superior, incluyendo universidades, institutos profesionales y centros de formación técnica, ofrecen varios miles de diferentes oportunidades de formación, conducentes a 360 grados y títulos, incluyendo bachilleratos, licenciaturas, títulos exclusivamente universitarios y sus especialidades, títulos profesionales y sus especialidades, títulos de ingenierías en ejecución y títulos técnicos. Este desarrollado sistema opera, en una gran medida, según las indicaciones que extrae del mercado, donde estas instituciones compiten para atraer a los alumnos.

En lo personal, he estado muy vinculado al ámbito educacional, primero como estudiante graduado de economía de la educación y luego como investigador. Creo, sin embargo, que mi opinión hoy está también influida por experiencias posteriores de gestión universitaria, como autoridad universitaria en lo académico y administrativo, por mi vinculación más reciente con el desarrollo de una relevante universidad privada y por una ya larga experiencia empresarial. Desde esta formación y experiencias, y reconociendo lo valioso que es el desarrollo de nuestro sistema educacional, me referiré en este trabajo a algunos aspectos que me parecen importantes. 
El primero toca a la natural preocupación de quienes tienen responsabilidad en el funcionamiento del sistema educacional, por la existencia de eventuales carencias o excesos en un medio esencialmente libre. A este respecto enfatizaré la necesidad de resistir la tentación de intervenir el sistema a través de la imposición arbitraria de criterios cuantitativos o cualitativos.

En segundo término, discuto el sentido en que me gustaría ver evolucionar el sistema de educación superior-siempre en un contexto de mercado- en cuanto a estructuración de los estudios de ese nivel, incluyendo aspectos metodológicos y de contenidos de la enseñanza.

Por último, indico mi preocupación por el negativo impacto sobre la calidad de la educación superior que podría estar teniendo la canalización directa, no competitiva, de parte de los recursos estatales que financian educación superior, e incluso del sistema de Aporte Fiscal Indirecto (AFI), si se le compara con un esquema universal de acceso al financiamiento de esos estudios.

\section{UN SISTEMA DE EDUCACIÓN SUPERIOR FLEXIBLE Y ADAPTABLE A NUEVAS DEMANDAS}

En un mundo sujeto a acelerados cambios científicos, tecnológicos e institucionales, encuentro valioso contar con un sistema de educación superior capaz de permitir constantes adaptaciones educacionales. Por eso en otras oportunidades he manifestado mis preferencias por un sistema muy libre, donde la demanda espontánea por programas de estudios específicos estimulase la respuesta adecuada de las entidades correspondientes.

Desde esta perspectiva de la flexibilidad del sistema para generar las respuestas educacionales correctas ante nuevos escenarios, me ha parecido siempre poco confiable un dirigismo educacional, orientado a garantizar que en cantidad o calidad la oferta educacional responda a una cierta noción de los "requerimientos" nacionales en materia de educación superior. Así, pienso que un intento de orientar o regular la oferta educacional arriesga una sobresimplificación peligrosa de las muchas dimensiones tras la demanda por educación superior, 
dimensiones que se expresan en una gran heterogeneidad en la oferta. Negar esta diversidad, normar y estandarizar, probablemente conduzca a serias ineficiencias.

\section{La diversidad tras una aparente homogeneidad}

En esta línea de pensamiento, considero importante reconocer y respetar el hecho de que, bajo un mismo nombre, se están ofreciendo hoy contenidos muy diversos, que probablemente responden a distintas demandas. Por ejemplo, tras las muchas decenas de programas conducentes a títulos en ingeniería comercial, los hay de amplitud e intensidad muy diferentes. Algunos están orientados a lograr una formación muy sólida en disciplinas básicas en torno a las cuales se estructura esta profesión, como son economía, matemáticas o estadística. Otros optan por un enfoque más aplicado. Algunos suponen completa concentración del estudiante por cinco años de estudios muy intensos; otros exigen solo dedicación parcial, compatible con alguna labor remunerada ejercida en paralelo.

Son opciones diversas, que responden a necesidades diversas, y que involucran recursos disímiles. Los profesores y los alumnos en los respectivos programas se desempeñan en forma diferente. Son alternativas educacionales distintas, con costos y objetivos diferenciados.

Ante esta realidad debemos ser cautelosos. Posiblemente exista algo de desinformación que, en algunos casos, lleve a entender como semejante lo que es diferente. Pero es innegable que la mayor parte de las diferencias son percibidas como tales y aceptadas por todos los involucrados. Muy probablemente la diversidad que se presenta y acepta bajo un mismo nombre refleja una gran diversidad de demandas educacionales y de recursos humanos e intelectuales dedicados a la educación superior.

¿Limitar, regular o clasificar?

Si aceptamos que hay esa diversidad tras un mismo nombre, ¿qué actitud cabe para perfeccionar el proceso de educación superior? Luego de hacerse cargo de que bajo un mismo nombre existe gran diversidad, parece evidente que tiene poco sentido discutir si es excesiva o 
insuficiente la matrícula total en ingeniería comercial, tratada esta como algo homogéneo. Tampoco me parece fácil diferenciar los títulos profesionales y ordenarlos en subgrupos. Quizás sí haya beneficios en establecer la obligatoriedad de informar adecuadamente sobre el real contenido de los programas educacionales, materia en la cual, nuevamente, cabe una seria discusión para determinar qué elementos son los significativos al momento de informar para permitir la diferenciación.

En relación con lo complejo que es clasificar y comparar, es ilustrativa una anécdota, que circula en forma oral, sobre lo que las universidades de Harvard y de Chicago destacan como diferencia relevante entre sus respectivas escuelas de posgrados en administración de empresas. Chicago, ironizando, indica que el enfoque de "casos" con el que se apoya la enseñanza en Harvard es inútil, porque la probabilidad de encontrarse uno de esos casos en la vida real es remota y... jocosamente agrega, que si alguien se llegara a topar con un caso descrito, lo más probable es que ya se hubiese olvidado la respuesta. Harvard, en tanto, refuta diciendo que el enfoque más analítico que se prefiere en Chicago prepara... buenos analistas, pero que para manejar bien una empresa se necesita alguien formado en términos más prácticos, como se hace en Harvard.

A juicio de estas grandes universidades, más allá del número de profesores con estudios de doctorado, de la infraestructura, de las bibliotecas, de los programas de intercambio, lo que haría más ventajoso un programa sería la forma de apreciar el rol del ejecutivo en la empresa y la traducción de esa visión en un enfoque metodológico para la enseñanza. El número de volúmenes en biblioteca, o los metros cuadrados construidos, son indicadores fáciles de recopilar y tabular pero, desgraciadamente, la clasificación útil, al menos como lo refleja la pugna Chicago-Harvard, debería incluir también criterios bastante más complejos.

La información debe estar disponible

Lo interesante es constatar que en el caso Chicago-Harvard las diferencias, que ambas escuelas enfatizan, que profesores y alumnos reconocen y que las empresas que contratan a los egresados aprecian 
como algo muy relevante, no han conducido a discutir si en ambos casos corresponde un mismo título o grado académico. Las diferencias existen y son reconocidas como importantes, sin que se considere necesario reflejarlas en lo formal.

Pero también es fundamental anotar que ambas universidades, junto con este esfuerzo por poner en discusión aspectos metodológicos muy complejos, pero que estiman que sus alumnos deben conocer, otorgan la más completa información sobre todos los aspectos físicos de las escuelas, profesores, programas de estudio y hasta contenido de los exámenes requeridos en cada curso.

Me parece que no hay que arriesgar fuertes ineficiencias en el sistema educacional introduciendo limitaciones a la matrícula según áreas o rigidizando los contenidos programáticos posibles de asociar a un cierto título o grado académico. Tampoco creo que se deban gastar recursos siempre escasos en diferenciar y clasificar en forma muy taxativa. Sí creo en la necesidad de generar la más amplia información sobre todos los contenidos programáticos y todo el quehacer de las distintas unidades académicas de educación superior. Es esperable que, también en lo relativo a información, el mercado producirá una presión creciente sobre las instituciones, cuya reacción debiera ser explicar mejor el verdadero contenido de sus programas.

\section{II. ¿CÓMO EDUCAR?}

He querido presentar primero una visión favorable a un sistema educacional muy libre, que deriva de mi convencimiento de que la eficiencia de ese sistema estará muy ligada a su capacidad de adaptación y evolución en un mundo cambiante, donde las demandas educacionales varían continuamente. En este contexto de apreciación por un esquema de libertad y de respeto por lo que el mercado pueda hacer surgir, me permitiré algunas reflexiones sobre el sentido en que personalmente considero debería evolucionar la educación superior chilena para otorgar una mejor formación.

En Chile existe una fuerte tradición educacional orientada a la formación de profesionales, a través de programas que se nutren de 
disciplinas básicas y que integran también elementos de conocimiento práctico, para producir un alto grado de especialización.

La misma visión de un mundo vertiginosamente cambiante en lo científico, tecnológico, económico y político, que me induce a preferir un sistema educacional con una máxima agilidad para adaptarse, me lleva a lamentar la relativa estrechez de visión que tiende a fomentar un sistema profesionalizante en la educación superior chilena.

Por comparación, me parece que el sistema estructurado en torno a grados académicos, propio de la tradición sajona, permite a los estudiantes la exploración de intereses más amplios, el mejor desarrollo de capacidades analíticas y, finalmente, en la etapa de estudios graduados, la misma concentración en temas muy especializados y en herramientas "profesionales" que caracterizan toda la etapa de formación universitaria en el sistema chileno.

En nuestro país los jóvenes deben elegir a los dieciocho años la profesión de su vida. Luego, a partir del primer año en la universidad, profundizan en un área del conocimiento, a través de aproximaciones sucesivas a una disciplina y su aplicación a ciertos temas, primero en nivel introductorio y progresivamente en niveles de mayor rigurosidad. Todo esto logra una gran especialización, pero conlleva una menor amplitud de visión, menores conocimientos de disciplinas científicas diferentes a las de la propia especialización, menor exposición a otros conocimientos que, inútiles desde la perspectiva de su área de interés profesional, serían una gran contribución a su capacidad global de apreciar y ponderar elementos de juicio.

No hay dudas de que la especialización y el conocimiento acabado son necesarios. Pero en el nivel superior de organización en la empresa, la universidad o el gobierno, se requiere también una visión más global, una cierta capacidad de entender algunos aspectos claves en la operatoria de diversos sistemas. Ahí se valora una mente que haya estado expuesta a comprender la forma en que se piensa en disciplinas no relacionadas con las de la propia especialidad. El deslumbramiento con la informática, por ejemplo, que llevó recientemente a grandes pérdidas de recursos, parece tener que ver con decisiones tomadas por especialistas en administración de empresas con poca sensibilidad 
y comprensión de fenómenos tecnológicos y por expertos en informática que no supieron reconocer las limitaciones que imponen los mercados. Los compartimentos estancos y el conocimiento excesivamente especializado pueden conducir a decisiones tomadas en forma temeraria, cuando no se pueden medir las consecuencias, o a una excesiva cautela.

Afortunadamente, existen muchas personas con esa capacidad de entender más de un sistema y, por ende, de lograr extraer de los especialistas lo requerido para el tratamiento de un problema concreto. Esas personas pueden liderar con menor riesgo de equivocarse.

No me parece que la ventaja de conocer más de un modo de pensar, de haber estado expuesto a más de una disciplina, solo se presente en los niveles de dirección de las grandes empresas o instituciones de gobierno. En diferente medida, posiblemente, la capacidad de captar mejor lo relevante que ocurre en otras áreas puede mejorar las decisiones de cualquier persona. De una u otra forma, abogados, empresarios, cientistas políticos, ingenieros, están expuestos a un mundo cambiante donde los problemas tienen múltiples aristas, cuya comprensión requiere de algún grado de interacción entre diferentes modos de pensar científico y de un bagaje cultural amplio. Piénsese, por ejemplo, en las capacidades y conocimientos que requiere identificar y desarrollar el tipo de intercambios, negocios o asociaciones que puedan generarse como consecuencia de un tratado de libre comercio con la Unión Europea o con Estados Unidos.

No se trata de negar valor a la especialización. Por el contrario, se trata de abrirle más espacio a la contribución técnica del especialista apreciando adecuadamente las posibilidades que se van abriendo en un mundo globalizado, con fuertes cambios tecnológicos, con amplia interacción entre diferentes culturas.

Lo que postulo es que la capacidad profesional de los individuos se verá potenciada por una exposición suficiente al aporte de otras disciplinas, sean estas la literatura, la filosofía, la biología o las matemáticas, según la inquietud de cada cual. Creo que el graduado de un programa de máster o de doctorado en economía en una universidad americana, que primero egresó de un college americano, 
donde estudió filosofía, matemáticas y algo de biología, simplemente ha tenido una mejor educación que un ingeniero comercial chileno que luego obtuvo su maestría o doctorado en esa misma universidad.

Las ventajas del sistema educacional estructurado en torno a grados académicos, con un bachillerato inicial donde el alumno puede explorar diversas disciplinas y estudios graduados posteriores, han sido ampliamente discutidas en nuestro país. Se han aducido a favor de este esquema diversos elementos, a saber: la posibilidad para el alumno de postergar la selección de su área específica hasta conocer mejor sus propias potencialidades así como diversas disciplinas; la posibilidad para el joven de elegir por sí mismo su carrera, una vez que esté más maduro, en contraste con una decisión temprana en el sistema actual que es muy influida por los padres, y, por último, permitir al estudiante adquirir mayor madurez mientras adquiere conocimientos más amplios que a través de cursos introductorios de su misma disciplina básica, con menor contenido.

Estos análisis han conducido a que algunas universidades chilenas estén adoptando el sistema del bachillerato. Pensamos que esto es algo muy positivo, en la medida en que dará mejores herramientas para la vida profesional de sus alumnos. Esperaríamos que la ventaja de una formación amplia lleve a muchos estudiantes a preferir las universidades que optaron por este enfoque, lo que hará al sistema evolucionar en tal sentido.

Con todo, percibo una cierta lentitud en la adopción de este esquema. Creo que al respecto ocurre lo mismo que con muchas marcas o etiquetas, que pueden llegar a tener mucha fuerza. Es el caso de la estructura profesionalizante del sistema educativo chileno. Las profesiones tradicionales y el esquema de formación que conduce a ellas tienen un bien ganado prestigio. Lo conservador y cauto, entonces, en opinión de apoderados y jóvenes, es seguir el camino conocido.

Así, las carreras tradicionales atraen a los mejores alumnos. Los mejores alumnos, a su vez, representan el mayor atractivo de las carreras. Hay una suerte de círculo vicioso o de inercia a favor de lo más convencional. En un sistema en que, a través del AFI, se apoya de modo indirecto la demanda libremente expresada, las universidades 
siguen la voluntad soberana de los estudiantes, la que aún tiende a reforzar a un sistema subóptimo.

La situación descrita entorpece el cambio hacia una modalidad de educación superior que parece más adecuada. Como economista formado en temas monetarios y relativos a educación, veo una analogía entre la lenta evolución del sistema educacional y la subsistencia de dineros nacionales de pésima calidad, permanentemente desvalorizados por la inflación, pero que persisten por la simple razón de que, para sustituirlos por uno mejor, es necesario el cambio simultáneo al nuevo dinero de una gran masa de público que lo haga utilizable como medio de pago. Ese proceso simultáneo es difícil, lento y eventualmente nunca llega. Esperemos que en el caso de nuestro sistema de educación superior ese cambio, aunque paulatino, ocurra en un grado relevante.

\section{ROL DEL ESTADO PARA MEJORAR LA CALIDAD DE LA EDUCACIÓN SUPERIOR}

Habiendo constatado mi preferencia por un sistema que amplíe el rango de conocimientos que se transmiten al estudiante en sus primeros años, para concentrar la especialización en las etapas finales, debo expresar, una vez más, que no creo que el Estado deba usar su músculo financiero para inducir una evolución más acelerada en ese sentido. Estoy a favor de un sistema según el cual el apoyo estatal no discrimine entre carreras, universidades o enfoques educacionales, sino que, simplemente, subsidie la demanda respetando las preferencias de quienes adquieren los servicios educacionales.

La situación contemporánea de intervención estatal en el funcionamiento de la educación superior no es perfecta. Ciertas modificaciones permitirían, en mi opinión, avanzar para hacer más neutro el apoyo del Estado al desarrollo del sistema de educación superior, al mismo tiempo que corregir deficiencias de calidad donde éstas existan.

Estoy convencido de que el subsidio directo y no condicionado a la oferta, el sostenimiento con recursos fiscales de una cierta capacidad 
de ofrecer educación superior, eximiendo total o parcialmente a las instituciones favorecidas de la necesidad de atraer su propio financiamiento, lleva a una menor calidad del servicio educacional producido. El acceso seguro a los recursos exime de la competencia y afecta negativamente la calidad del producto que se genera, se trate de docencia o de investigación. La menor calidad tiende a deteriorar el nivel del alumnado. El intento de atraer jóvenes, a pesar de una menor calidad, conduce a reducir los aranceles cobrados a los estudiantes. Profesores con la estabilidad laboral que deriva del subsidio asegurado, junto a alumnos que hacen un esfuerzo financiero menor para seguir sus estudios, llevan a un ambiente de baja exigencia, de frustración, alargamiento innecesario de la duración de los estudios y, en definitiva, a un mal uso de los recursos públicos y privados.

Me parece que la mejor alternativa es desarrollar un buen sistema de financiamiento para que los estudiantes cancelen los costos efectivos de su educación superior. Es bien conocido el análisis que indica que la educación superior de buena calidad mejora la capacidad de generar ingresos del estudiante, de manera que permite cancelar la deuda contraída para estudiar. Un sistema que se financia sobre la base de aranceles cancelados por los alumnos fomenta un ambiente de eficiencia, seriedad y esfuerzo en cada institución educacional. Al mismo tiempo, en la medida en que el alumno percibe el costo de su educación, además de exigir enseñanza de calidad, decide qué estudiar y por cuánto tiempo, con la debida consideración a las posibilidades de mejoramiento de ingreso que le reportará ese estudio. Esto ayuda a evitar el exceso de profesionales en ciertas áreas. Por último, debe anotarse que, desde un punto de vista de equidad distributiva, los subsidios a la educación superior favorecen a sectores que ya tienen más ingresos o los tendrán al egresar, lo que los hace relativamente regresivos.

Por cierto que, como complemento de un sistema donde los estudiantes financian su educación superior, con recursos propios o con préstamos, se puede canalizar apoyo estatal para fines específicos, sean estos de tipo distributivo, de fomento a las artes, de subsidio a determinadas profesiones con "externalidades" sociales positivas (como podría ser el caso de las pedagogías, según algunos análisis) o de apoyo a determinadas investigaciones de interés para el país. 
Como segunda mejor alternativa, el sistema del AFI es atractivo. Tiene la ventaja de que no otorga recursos garantizados y libres de competencia, lo que preserva el dinamismo en las instituciones de educación superior, pero mantiene un subsidio importante para los alumnos. Parece ser que en la medida en que los alumnos pagan menos que el verdadero costo de su educación, tiende a debilitarse el esfuerzo, a alargarse innecesariamente la duración de los estudios y a generarse la sobreinversión en algunos tipos de educación, que luego se percibe como "exceso" de profesionales.

Un empuje resuelto en el sentido de ampliar y mejorar el acceso al crédito para la educación (que supone enfrentar adecuadamente el problema de las dificultades de cobranza de estos créditos), reduciendo al mismo tiempo los aportes directos e indirectos a las instituciones de educación superior, llevaría, a mi juicio, a un seguro mejoramiento en la calidad de la educación superior chilena y a una mayor equidad en el gasto del sector público. El Estado, con sus recursos, podría impulsar programas o áreas de estudio e investigación muy precisos, siempre a través de mecanismos que mantengan un ambiente competitivo y de excelencia en la docencia e investigación.

Créditos para el financiamiento de la educación superior

Hace un año se dio a conocer un proyecto de ley orientado a desarrollar mejores mecanismos de financiamiento para la educación superior. El proyecto tenía serias limitaciones. Básicamente, creaba una institución capitalizada por el Estado y las universidades para otorgar créditos a estudiantes universitarios, en conformidad a algún reglamento. Por esta vía -y ahí percibo su debilidad-, un colectivo de universidades partícipes en el sistema y el Estado serían codeudores solidarios de cada alumno. Me parece evidente que la creación de una especie de "clase" de profesionales con deudas con esta institución solo puede dificultar la cobranza posterior de los préstamos. Por otra parte, la asociación entre universidades y Estado para avalar créditos universitarios debilita la responsabilidad de cada institución particular por recuperar los recursos otorgados.

Una alternativa simple sería disponer de recursos estatales para aportar al fondo creado por cada universidad individual, para garantizar 
los préstamos a sus respectivos estudiantes que voluntariamente quieran otorgar los bancos comerciales. Las universidades, los alumnos y los bancos estarían entonces vitalmente interesados en la calidad de la educación impartida. Una educación de calidad abriría oportunidades laborales a los egresados, lo que evitaría pérdidas al Estado, a la universidad que avala y a los bancos. Cada universidad, con el apoyo no discriminatorio del Estado dispuesto a ser codeudor del alumno y de la universidad, buscaría abrir líneas de crédito bancario a sus estudiantes, solo en la medida en que ella misma está convencida del valor privado y social de los conocimientos que imparte.

He revisado aspectos relativos a la supervisión estatal del sistema de educación superior, a la lógica profesionalizante de la educación universitaria chilena, a la subsistencia de formas subóptimas de intervención estatal en el sistema de educación superior. Estos aspectos, en mi opinión, son muy importantes en la conformación de un sistema de educación sensible a los requerimientos del país y de los propios estudiantes. No me he referido al grande y complejo tema de los aspectos institucionales y de diversa índole que inciden en el desarrollo de la investigación. Hay algo profundamente estimulante en el ambiente que se respira en los grandes centros de investigación existentes en algunos países desarrollados; quienes han podido experimentar esa inmersión profunda en un mundo de ideas y de búsqueda apasionada de nuevas verdades, no pueden sino reconocer que es ahí donde se gestan las grandes transformaciones científicas y culturales del mundo. De ahí, la aspiración a que el sistema de educación superior chileno consolide y expanda su propia capacidad de desarrollar investigación rigurosa, vasta y capaz de orientar los esfuerzos materiales y espirituales de la nación. Ojalá la revista Calidad en la Educación tenga éxito en su esfuerzo por identificar los elementos capaces de distinguir a Chile por su excelencia en la educación superior y en la investigación en las diversas áreas del conocimiento. 